\title{
Very low levels of serum anti-Müllerian hormone as a possible marker for follicle growth in patients with primary ovarian insufficiency under hormone replacement therapy
}

\section{Yukiyo Kasahara}

Nagoya Daigaku

Satoko Osuka ( $\sim$ satokoosuka@med.nagoya-u.ac.jp )

Nagoya Daigaku https://orcid.org/0000-0002-4430-7982

Bayasula Bayasula

Nagoya Daigaku

Natsuki Nakanishi

Nagoya Daigaku

Tomohiko Murase

Nagoya Daigaku

Tomoko Nakamura

Nagoya Daigaku

Maki Goto

Nagoya Daigaku

Tomomi Kotani

Nagoya Daigaku

Akira Iwase

Gunma Daigaku

Fumitaka Kikkawa

Nagoya Daigaku

Research

Keywords: primary ovarian insufficiency, anti-Müllerian hormone, picoAMH, ultrasensitive assay, follicle growth

Posted Date: May 16th, 2020

DOI: https://doi.org/10.21203/rs.2.22083/v3 
License: (c) (i) This work is licensed under a Creative Commons Attribution 4.0 International License. Read Full License

Version of Record: A version of this preprint was published at Reproductive Sciences on July 31st, 2020. See the published version at https://doi.org/10.1007/s43032-020-00278-4. 


\section{Abstract}

Background: Patients with primary ovarian insufficiency (POI) occasionally present with follicle growth; however, it is difficult to predict exactly which cycles will show follicle growth. Serum anti-Müllerian hormone (AMH), a useful marker for ovarian reserve, is produced in early-stage follicles. Therefore, $\mathrm{AMH}$ levels should reflect the presence of small follicles, which are difficult to detect using ultrasonography, and may be useful as a marker for predicting follicle growth in patients with POI.

Methods: Using an ultrasensitive enzyme-linked immunosorbent assay (ELISA) kit, we found very low serum $\mathrm{AMH}$ levels in patients with $\mathrm{POI}$. We assessed the follicle growth of each patient during each cycle to clarify the usefulness of measuring serum AMH levels for predicting follicle growth in patients with POI under hormone replacement therapy (HRT).

Results: A total of 91 cycles of 19 patients with POI were analyzed in this study. Five patients presented with positive serum AMH levels during the observational periods, and all five experienced follicle growth. Only two of the 14 patients with negative serum AMH levels had follicle growth. Serum AMH and folliclestimulating hormone (FSH) levels in cycles with follicle growth were significantly higher than in cycles without follicle growth. The median serum AMH level $\left(2.77 \mathrm{pg} / \mathrm{mL} ; 25^{\text {th }}, 75^{\text {th }}\right.$ percentiles: $\left.0.0,9.64\right)$ in cycles with follicle growth was lower than the lower limit of detection of conventional AMH ELISA kits. The positive predictive value of positive serum $\mathrm{AMH}$ levels for follicle growth was higher than that of FSH $(<10 \mathrm{mlU} / \mathrm{mL})$.

Conclusions: Measurement of very low levels of serum AMH using picoAMH assays is useful for prediction of follicle growth in patients with POI under HRT conditions.

Trial registration: UMIN-CTR UMIN000029464. Retrospectively registered on April 4, 2018. https://upload.umin.ac.jp/cgi-open-bin/ctr/ctr_view.cgi?recptno=R000033550

\section{Background}

Primary ovarian insufficiency (POI) is defined as the development of hypergonadotropic hypogonadism before the age of 40 years (1)(2). POI is one of the causes of intractable infertility. Due to the current trend of women delaying having their first child until later in life, the number of patients with POI who desire children is increasing. Some patients with POI have follicle growth occasionally (3), although it is difficult to predict which cycles will show follicle growth. Currently, serum follicle-stimulating hormone (FSH) or estradiol (E2) levels are measured in the clinical setting to help predict follicle growth in patients with POI. Serum E2 levels were reported to be a useful predictive factor for ovarian function in patients with POI (4); however, an elevation of serum E2 levels is a result of follicle growth, and therefore, follicles are already growing in these conditions. Likewise, serum FSH levels are affected by hormonal therapy, and consequently, some cycles with low serum FSH levels did not show follicle growth even with ovarian stimulation due to prior human gonadotropin injections (5)(6). 
Thus, markers with higher predictive value are required even in these conditions, in order to avoid ineffective ovarian stimulations which result in not only economic, but also physical and psychosocial, burdens for the patients.

Among several markers for ovarian reserve, the serum level of anti-Müllerian hormone (AMH), which is produced by the granulosa cells of early-stage follicles, is considered to be one of the most informative markers for ovarian reserve (7)(8)(9). Measurement of AMH levels in the clinical setting has thus been used extensively in, for example, the prediction of ovarian response to controlled ovarian stimulation by gonadotropins, optimization of protocols in assisted reproductive technology, and assessment of ovarian toxicity in medical and surgical conditions (9)(10)(11)(12). In recent years, a new AMH ultrasensitive enzyme-linked immunosorbent assay (ELISA) kit, the picoAMH ELISA (MenoCheck picoAMH, Ansh Labs, Webster, TX USA) for the detection of very low serum AMH levels, has become available. The lower limit of detection (LoD) of the picoAMH ELISA is $3 \mathrm{pg} / \mathrm{mL}$. This is significantly lower than that of the Gen II AMH ELISA (Beckman Coulter Inc., Brea, CA USA), one of the most prevalent AMH assay kits, which has a LoD of $80 \mathrm{pg} / \mathrm{mL}$. We reported that measuring very low serum $A M H$, which is not detected by conventional ELISA kits, in patients with POI was useful for predicting true ovarian reserve (13).

In the current study, we investigated the usefulness of measuring very low levels of serum AMH using an ultrasensitive AMH assay kit, picoAMH, as the marker for follicle growth of a cycle in patients with POI under HRT conditions.

\section{Methods}

\section{Patients}

Patients with POI who underwent cyclic hormonal therapy between April 2013 and March 2019 were recruited for the study. Patients with POI are defined as those under 40 years of age who exhibit serum $\mathrm{FSH}$ levels $>40 \mathrm{mIU} / \mathrm{mL}$ on at least two different tests, and present with amenorrhea for more than three months in the absence of hormonal therapy. The cyclic hormonal therapies administered included conjugated equine estrogen (Premarin®; Pfizer Inc., New York, NY USA), 2 tablets/day for 10 days followed by an estrogen ( $0.05 \mathrm{mg}$ ethinylestradiol) and progestin ( $0.5 \mathrm{mg}$ norgestrel) combination (Planovar ${ }^{\circledR}$; Takeda Pharmaceutical Company Limited, Chuo-ku, Tokyo, Japan), 1 tablet/day for 11 days, or alternatively Premarin ${ }^{\circledR} 2$ tablets/day for 21 days with chlormadinone acetate (Lutoral ${ }^{\circledR} ;$ Fuji Pharma Co. Ltd., Chiyoda-ku, Tokyo, Japan), 2 tablets/day, for the last 11 days. Following a 21-day treatment with a given cyclic hormonal therapy protocol, patients were subjected to a seven-day withdrawal period before the next cyclic hormonal therapy was started. If serum E2 levels exceeded $30 \mathrm{pg} / \mathrm{mL}$, or serum FSH levels dropped to $<15 \mathrm{mIU} / \mathrm{mL}$, during the withdrawal period, the next cyclic hormonal therapy was suspended, and the follicles were monitored once or twice a week by ultrasonography. Follicle growth was defined as the presence of follicles with a mean diameter $>16 \mathrm{~mm}$. In some cases, human menopausal gonadotropins were administered for controlled ovarian stimulation. This study was 
approved by the ethical committee of Nagoya University Graduate School of Medicine, and informed consent was obtained from all patients.

\section{Hormonal measurements}

Blood samples were obtained during the drug withdrawal period of each patient. Serum was isolated using a centrifuge and separated from the whole blood samples, transferred to sterile polypropylene tubes, and stored at $-80^{\circ} \mathrm{C}$ until the assays were performed. The serum picoAMH concentrations were measured using the picoAMH ELISA kit, according to the manufacturer's instructions (14)(15) by the same operator. The dynamic range of the standard curve of the picoAMH was from 3.0 to $746 \mathrm{pg} / \mathrm{mL}$, and the LoD and limit of quantitation (LoQ) were $1.2 \mathrm{pg} / \mathrm{mL}$ and $3.9 \mathrm{pg} / \mathrm{mL}$, respectively, in accordance with the manufacturer's specifications. In this study, $0.00 \mathrm{pg} / \mathrm{mL}$ measured with the picoAMH was defined as an undetectable (negative) AMH. The absorbance measurements were performed using a microplate reader (ELx808 ${ }^{\mathrm{TM}}$ Absorbance Microplate Reader; BioTek Instruments, Inc., Winooski, VT USA). Concentrations of serum antithyroperoxidase antibody (TPOAb), antithyroglobulin antibody (TgAb), E2, and FSH were measured using enzyme-linked immunosorbent assay kits (Elecsys Anti-Tg, Anti-TPO; Roche Diagnostics, Rotkreuz, Switzerland; ARCHITECT ${ }^{\circledR}$ Estradiol, ARCHITECT ${ }^{\circledR}$ FSH; Abbott Laboratories, Abbott Park, IL USA). Serum TPOAb levels $>16 \mathrm{IU} / \mathrm{mL}$ and TgAb levels $>28 \mathrm{IU} / \mathrm{mL}$ were considered positive.

\section{Statistical analyses}

The data were analyzed using the Prism software (Prism 8; GraphPad Software, San Diego, CA USA), and EZR software (Version 1.40; Saitama Medical Center, Jichi Medical University, Saitama, Japan), the latter of which is a graphical user interface for $\mathrm{R}$ (The R Foundation for Statistical Computing, Vienna, Austria). More precisely, EZR is a modified version of R Commander designed to add statistical functions that are frequently used in biostatistics (16). Mann-Whitney $U$ tests were used to compare patient characteristics, including hormone levels, while Fisher's exact tests were applied to compare the proportions of groups. A $p$-value of $<0.05$ was considered to indicate the level of statistical significance.

\section{Results}

A total of 91 cycles of 19 patients with POI were analyzed in this study. Out of the total of 19 patients, four had histories of chemotherapy, three had a documented chromosomal abnormality, and 12 patients lacked a definitive cause for the POI. Out of these 12 patients, seven were positive for thyroid autoantibodies (TPOAb and/or TgAb). Table 1 shows the patient characteristics categorized by positive or negative serum AMH measured using the picoAMH kits. The median age of onset of POI did not differ between the negative and positive AMH groups. All of the patients with one or more AMH-positive cycles experienced follicle growth during the observation periods. In contrast, out of the group of patients who were $\mathrm{AMH}$-negative, only $14.3 \%$ experienced follicle growth (Table 1 ). 
For the purpose of evaluating $\mathrm{AMH}$ as a predictive factor for follicle growth of the cycle, we analyzed the serum levels of $\mathrm{AMH}$ and FSH and the follicle growth of each cycle. Of the total $91 \mathrm{cycles}, 14$ were positive for $\mathrm{AMH}$, and 14 cycles presented with follicle growth. The serum FSH levels of the cycles with follicle growth were significantly lower, and the serum $\mathrm{AMH}$ levels significantly higher, than those in cycles without follicle growth (Figure 1). The median serum FSH and AMH levels of cycles with follicle growth were $15.44 \mathrm{mIU} / \mathrm{mL}\left(25^{\text {th }}, 75^{\text {th }}\right.$ percentile: $\left.5.03,26.85\right)$ and $2.77 \mathrm{pg} / \mathrm{mL}\left(25^{\text {th }}, 75^{\text {th }}\right.$ percentile: 0.0 , 9.64), respectively. Out of the $14 \mathrm{AMH}$-positive cycles, nine presented with follicle growth, and five did not show follicle growth. However, of these five cycles, two were positive for AMH in the previous cycles. Serum FSH levels in the AMH-positive cycles were significantly lower than in the AMH-negative cycles (Table 2).

The positive predictive value (PPV) and negative predictive value (NPV) of AMH-positive serum for follicle growth of the cycle were 0.643 and 0.935 , respectively. At the same time, we assessed the usefulness of serum FSH levels in the prediction of follicle growth. When we set FSH levels $<10 \mathrm{mIU} / \mathrm{mL}$ as a predictor of follicle growth, the PPV of FSH was 0.250 and the NPV was 0.873 . These results indicate that AMHpositive serum during the withdrawal period is superior to low levels of serum FSH as a predictor of follicle growth in the cycle.

\section{Discussion}

In this study, we demonstrated that detection of very low serum AMH levels in patients with POI can be used as a marker for predicting follicle growth of the current cycle. In patients with POI, it has been reported that the possibility of spontaneous ovulation is about $4 \%$ during a cycle (3); however, it is difficult to predict the exact cycle which will present with follicle growth. Gonadotropin therapy with exogenous estrogen is one of the most common strategies for the induction of follicle growth in patients with POI (17)(6)(4). It has been reported that ovulation only occurred in patients with lower serum FSH (< $15 \mathrm{IU} / \mathrm{mL}$ ) after estrogen therapy (6), resulting in an ovulation rate of $32 \%$ with this therapy, higher than the spontaneous ovulation rate of patients with POI. However, $70 \%$ of the patients who underwent ovarian stimulation did not achieve ovulation.

In the current study, serum FSH levels in cycles with follicle growth were significantly lower than those in cycles without follicle growth. However, the PPV and NPV of serum $\mathrm{FSH}<10 \mathrm{mlU} / \mathrm{mL}$ as a prediction marker for follicle growth were 0.25 and 0.837 , respectively, resulting in $75 \%$ of the cycles with lower $\mathrm{FSH}$ levels not presenting with follicle growth. In contrast, the PPV and NPV of AMH-positive serum were 0.643 and 0.935 , respectively, significantly higher than those of low levels of serum $\mathrm{FSH}(<10 \mathrm{mIU} / \mathrm{mL}$ ). Moreover, the median serum AMH level of cycles with follicle growth was $2.77 \mathrm{pg} / \mathrm{mL}\left(25^{\text {th }}, 75^{\text {th }}\right.$ percentile: $0.0,9.64$ ), an undetectable level using the Gen II AMH ELISA (LoD: $80 \mathrm{pg} / \mathrm{mL}$ ) or automated assay systems such as the Elecsys AMH (LoD: $10 \mathrm{pg} / \mathrm{mL}$, Roche Diagnostics) and the Access AMH (LoD: 20 pg/mL, Beckman Coulter Inc.) (18)(15). 
There were five cycles presenting with follicle growth which had AMH-negative serum. Of these five cycles, two were AMH-positive in the previous cycle. Additionally, patients who had positive AMH levels one or more times presented with follicle growth during the observational periods. These results indicate that the measurement of very low serum AMH levels was useful for predicting follicle growth in patients with POI.

We acknowledge that there were several limitations to this study. Firstly, the numbers of patients and cycles included in this study were small. However, the frequency of ovulation of the patients and the frequency of cycles were $3.7 \%$ and $1.5 \%$, respectively, and these rates were similar to those reported in a previous study (3). Secondly, an automated assay system for picoAMH is not presently available, and consequently, it is difficult to get same-day results of picoAMH testing. Since serum AMH levels are not affected by hormonal status (19)(20), clinicians may measure AMH serum levels during hormonal therapy, or several days before the withdrawal periods, in order to predict follicle growth. Thirdly, the results of this study are limited to the prediction of follicle growth, and all seven patients who exhibited follicle growth in this study have failed to achieve pregnancy. Previous reports indicated that the median serum AMH level of patients with successful IVF was 837 (range: 141.6-2750) pg/mL (21), and live birth rate was significantly lower in women with $A M H \leq 0.1 \mathrm{ng} / \mathrm{mL}$ (22). Consequently, it cannot be declared that detection of $\mathrm{AMH}$ by the picoAMH kit in POI patients raises the probability of successful IVF or pregnancy. Fourthly, donor oocytes are available for patients with POI in some countries. However, there are still difficulties regarding accessing oocyte donations in some countries. Moreover, in vitro fertilization (IVF) with donor oocytes has some issues, such as the high expense and concerns regarding genetics. In addition, some reports have suggested that patients with spontaneous POI have an increased risk of perinatal complications, such as pregnancy-induced hypertension and postpartum hemorrhage, when they become pregnant using IVF with oocyte donation (23)(24). Therefore, the availability and feasibility of IVF with autologous oocyte could be significant for patients with POI.

In 2018, the U.S. Food and Drug Administration permitted marketing of the picoAMH ELISA diagnostic test as an aid in the determination of a patient's menopausal status (25). In addition to predicting menopausal status, the picoAMH ELISA kits may have significance for patients with POI who desire to have children.

\section{Conclusion}

In conclusion, we demonstrated the usefulness of very low levels of serum AMH as a marker for follicle growth in patients with POI. Further studies with larger numbers of patients are expected to demonstrate the benefits of measuring picoAMH in patients with POI.

\section{Declarations}

\section{Ethics approval and consent to participate}


The study was approved by the ethical committee of Nagoya University Hospital (approval reference number 422), registered as a clinical trial (UMIN000029464) on April 4, 2018, and informed consent was obtained from all patients prior to enrollment. All procedures were performed according to the Declaration of Helsinki regarding human experimentation.

\section{Consent for publication}

Not applicable.

\section{Availability of data and materials}

The datasets analyzed during the current study are available from the corresponding author on reasonable request.

\section{Competing interests}

The authors declare no potential conflicts of interest with respect to the research, authorship, and/or publication of this article.

\section{Funding}

The authors received no financial support for the research, authorship, and/or publication of this article.

\section{Authors' contributions}

YK performed the data analysis and the writing of the manuscript. SO analyzed and interpreted the data. Bayasula measured serum levels using the picoAMH assays. Data were collected and analyzed by TM, $\mathrm{NN}$, and TN. Interpretation of the data was done by MG, TK, Al, and FK. All authors read and approved the final manuscript.

\section{Acknowledgements}

Not applicable.

\section{Abbreviations}

POI: primary ovarian insufficiency

HRT: hormone replacement therapy

FSH: follicle-stimulating hormone

E2: estradiol 
AMH: anti-Müllerian hormone

TPOAb: antithyroperoxidase antibody

TgAb: antithyroglobulin antibody

ELISA: enzyme-linked immunosorbent assay

PPV: positive predictive value

NPV: negative predictive value

IVF: in vitro fertilization

\section{References}

1. Nelson LM. Primary ovarian insufficiency. N Engl J Med. 2009 Feb;360(6):606-14.

2. De Vos M, Devroey P, Fauser BC. Primary ovarian insufficiency. Lancet. 2010 Sep;376(9744):911-21.

3. Nelson LM, Anasti JN, Kimzey LM, Defensor RA, Lipetz KJ, White BJ, et al. Development of luteinized graafian follicles in patients with karyotypically normal spontaneous premature ovarian failure. $\mathrm{J}$ Clin Endocrinol Metab. 1994 Nov;79(5):1470-5.

4. Miyazaki K, Miki F, Uchida S, Masuda H, Uchida H, Maruyama T. Serum estradiol level during withdrawal bleeding as a predictive factor for intermittent ovarian function in women with primary ovarian insufficiency. Endocr J. 2015;62(1):93-9.

5. Surrey ES, Cedars MI. The effect of gonadotropin suppression on the induction of ovulation in premature ovarian failure patients. Fertil Steril. 1989 Jul;52(1):36-41.

6. Tartagni M, Cicinelli E, De Pergola G, De Salvia MA, Lavopa C, Loverro G. Effects of pretreatment with estrogens on ovarian stimulation with gonadotropins in women with premature ovarian failure: a randomized, placebo-controlled trial. Fertil Steril. 2007 Apr;87(4):858-61.

7. Broer SL, Broekmans FJM, Laven JSE, Fauser BCJM. Anti-Müllerian hormone: ovarian reserve testing and its potential clinical implications. Hum Reprod Update. 2014 Sep-Oct;20(5):688-701.

8. Iwase A, Osuka S, Goto M, Murase T, Nakamura T, Takikawa S, et al. Clinical application of serum anti-Müllerian hormone as an ovarian reserve marker: A review of recent studies. J Obstet Gynaecol Res. 2018 Jun;44(6):998-1006.

9. Visser JA, Schipper I, Laven JSE, Themmen APN. Anti-Müllerian hormone: An ovarian reserve marker in primary ovarian insufficiency. Nat Rev Endocrinol. 2012 Jan;8(6):331-41.

10. Iwase A, Nakamura T, Kato N, Goto M, Takikawa S, Kondo M, et al. Anti-Müllerian hormone levels after laparoscopic cystectomy for endometriomas as a possible predictor for pregnancy in infertility treatments. Gynecol Endocrinol. 2016;32(4):293-7. 
11. Iwase A, Nakamura T, Osuka S, Takikawa S, Goto M, Kikkawa F. Anti-Müllerian hormone as a marker of ovarian reserve: What have we learned, and what should we know? Reprod Med Biol. 2016 Nov;15(3):127-36.

12. Saito A, Iwase A, Nakamura T, Osuka S, Bayasula, Murase T, et al. Involvement of mesosalpinx in endometrioma is a possible risk factor for decrease of ovarian reserve after cystectomy: a retrospective cohort study. Reprod Biol Endocrinol. 2016 Oct 28;14(1):72.

13. Iwase A, Osuka S, Nakamura T, Kato N, Takikawa S, Goto M, et al. Usefulness of the ultrasensitive anti-Müllerian hormone assay for predicting true ovarian reserve. Reprod Sci. 2016 Jun;23(6):75660.

14. Iwase A, Osuka S, Nakamura T, Kato N, Takikawa S, Goto M, et al. Usefulness of the ultrasensitive anti-Müllerian hormone assay for predicting true ovarian reserve. Reprod Sci. 2016 Jun;23(6):75660.

15. Su HI, Sammel MD, Homer MV, Bui K, Haunschild C, Stanczyk FZ. Comparability of anti-Müllerian hormone levels among commercially available immunoassays. Fertil Steril. 2014 Jun;101(6):176672.e1.

16. Kanda Y. Investigation of the freely available easy-to-use software "EZR" for medical statistics. Bone Marrow Transplant. 2013 Mar;48(3):452-8.

17. Check JH, Nowroozi K, Chase JS, Nazari A, Shapse D, Vaze M. Ovulation induction and pregnancies in 100 consecutive women with hypergonadotropic amenorrhea. Fertil Steril. 1990 May;53(5):811-6.

18. Li HWR, Wong BPC, Ip WK, Yeung WSB, Ho PC, Ng EHY. Comparative evaluation of three new commercial immunoassays for anti-Müllerian hormone measurement. Hum Reprod. 2016 Dec;31(12):2796-802.

19. Streuli I, Fraisse T, Pillet C, Ibecheole V, Bischof P, de Ziegler D. Serum anti-Müllerian hormone levels remain stable throughout the menstrual cycle and after oral or vaginal administration of synthetic sex steroids. Fertil Steril. 2008; Aug;90(2): 395-400.

20. Li HWR, Wong CYG, Yeung WSB, Ho PC, Ng EHY. Serum anti-Müllerian hormone level is not altered in women using hormonal contraceptives. Contraception. 2011 Jun;83(6):582-5.

21. Burks HR, Ross L, Opper N, Paulson E, Stanczyk FZ, Chung K. Can highly sensitive antimüllerian hormone testing predict failed response to ovarian stimulation? Fertil Steril. 2015;104(3):643-8.

22. Reijnders IF, Nelen WLDM, Inthout J, Van Herwaarden AE, Braat DDM, Fleischer K. The value of AntiMüllerian hormone in low and extremely low ovarian reserve in relation to live birth after in vitro fertilization. Eur J Obstet Gynecol Reprod Biol. 2016; 200:45-50.

23. Söderström-Anttila V, Tiitinen A, Foudila T, Hovatta O. Obstetric and perinatal outcome after oocyte donation: comparison with in-vitro fertilization pregnancies. Hum Reprod. 1998 Feb;13(2):483-90.

24. Abdalla HI, Billett A, Kan AKS, Baig S, Wren M, Korea L, et al. Obstetric outcome in 232 ovum donation pregnancies. Br J Obstet Gynaecol. 1998 Mar;105(3):332-7. 
25. FDA news release: FDA permits marketing of a diagnostic test to aid in the determination of menopausal status. 24 Oct 2018. https://www.fda.gov/news-events/press-announcements/fdapermits-marketing-diagnostic-test-aid-determination-menopausal-status Accessed 15 Jan 2020.

\section{Tables}

Table 1. Comparison According to Serum AMH in patients with POI.

\begin{tabular}{lcccc}
\hline Variable & Overall $(\mathrm{n}=$ & AMH-negative $(\mathrm{n}=$ & AMH-positive $(\mathrm{n}=$ & $p$-value \\
& $19)$ & $14)$ & $5)$ & $\mathrm{b}$ \\
\hline Age at onset of POI (years) & $17.0(15.0$, & $16.0(14.0,20.0)$ & $22.0(17.0,27.0)$ & $0.876^{\mathrm{c}}$ \\
& $23.0)$ & & & \\
Age at first observation period & $25.2(23.4$ & $24.8(20.7,31.2)$ & $27.6(24.7,33.4)$ & 0.3913 \\
(years) & $30.8)$ & & & $\mathrm{c}$ \\
\hline Serum FSH $^{\text {a }}(\mathrm{mIU} / \mathrm{mL})$ & $46.4(22.7$ & $46.4(33.1,75.3)$ & $66.2(13.2,78.5)$ & 0.9644 \\
& $70.5)$ & & $5(100)$ & 0.0018 \\
\hline Follicle growth, $\mathrm{n}(\%)$ & $7(37)$ & $2(14)$ & & $\mathrm{d}$ \\
\hline
\end{tabular}

AMH, anti-Müllerian hormone; POI, primary ovarian insufficiency; FSH, follicle-stimulating hormone AMH-positive, patients with one or more AMH-positive cycles

AMH-negative, patients without AMH-positive cycles

Follicle growth, number of patients with a history of follicle growth in one or more of the observation periods

Values are presented as median $\left(25^{\text {th }}, 75^{\text {th }}\right.$ percentile!

a Serum FSH levels at first observation period.

$\mathrm{b}$ Compare AMH-positive and negative groups.

${ }^{\mathrm{c}}$ Mann-Whitney $U$ test

d Fisher's exact test

Table 2. Serum AMH and FSH and follicle growth in AMH-positive or negative cycles. 


\begin{tabular}{lccc}
\hline Variable & $\begin{array}{c}\text { AMH-negative } \\
(\mathrm{n}=77)\end{array}$ & $\begin{array}{c}\text { AMH-positive } \\
(\mathrm{n}=14)\end{array}$ & $p$-value \\
\hline Serum FSH $(\mathrm{mIU} / \mathrm{mL})$ & $33.0(20.4-49.0)$ & $10.0(5.0-26.6)$ & $0.0058^{\mathrm{a}}$ \\
Serum AMH $(\mathrm{pg} / \mathrm{mL})$ & $0.0(0.0-0.0)$ & $7.7(4.6-22.3)$ & $<0.0001^{\mathrm{a}}$ \\
\hline Follicle growth, $\mathrm{n}(\%)$ & $5(6)$ & $9(64)$ & $<0.0001^{\mathrm{b}}$ \\
\hline
\end{tabular}

AMH, anti-Müllerian hormone; FSH, follicle-stimulating hormone

Values are presented as median $\left(25^{\text {th }}, 75^{\text {th }}\right.$ percentile [

a Mann-Whitney $U$ test

${ }^{\mathrm{b}}$ Fisher's exact test

\section{Figures}
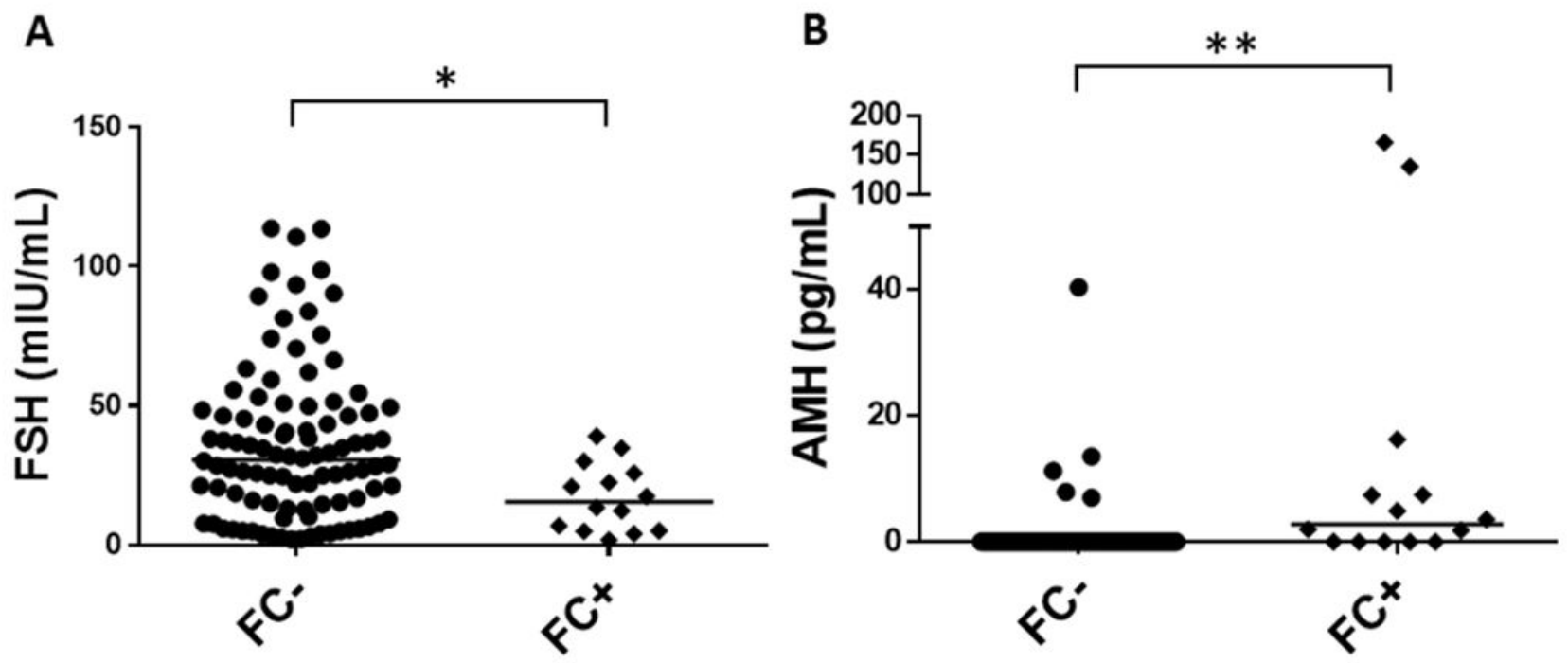

Figure 1

Serum FSH (A) and AMH (B) levels of cycles with or without follicle growth. Serum FSH levels were significantly lower and serum AMH levels were significantly higher in the cycles with follicle growth. *, $\mathrm{p}<$ $0.01, * \star, p<0.0001$, by Mann-Whitney U test. Bars represent medians. FC, Follicle growth. 